\title{
ATLAS Electron and Photon Trigger
}

\section{Joaquin Hoya, on behalf of the ATLAS Collaboration*}

Universidad Nacional de La Plata (Argentina) and Université de Genève (Suisse)

E-mail: joaquin.hoya@cern.ch

\begin{abstract}
ATLAS electron and photon triggers covering transverse energies from $5 \mathrm{GeV}$ to several $\mathrm{TeV}$ are essential to record signals for a wide variety of physics: from the Standard Model processes to searches for new phenomena. To cope with ever-increasing luminosity and more challenging pile-up conditions at a centre-of-mass energy of $13 \mathrm{TeV}$, the trigger selections need to be optimized to control the rates and keep efficiencies high. The ATLAS electron and photon trigger in Run-2 is presented, including new techniques developed to maintain their high performance even in high pile-up conditions as well as the first efficiency measurements from the 2018 data taking.
\end{abstract}

Sixth Annual Conference on Large Hadron Collider Physics (LHCP2018)

4-9 June 2018

Bologna, Italy

${ }^{*}$ Speaker. 


\section{Introduction}

The trigger system [1] of the ATLAS detector [2] is the responsible for deciding which events are saved, for later analysis, during the data taking. In particular, electrons and photons are present in many interesting final states studied by different physics analyses like the Standard Model (SM) measurements and Beyond the Standard Model (BSM) searches, including Supersymmetry (SUSY) and exotics models. Triggering on electrons and photons is then a key task of the acquisition system.

The ATLAS trigger is a two-level system designed to reduce the average event rate recording to $\sim 1 \mathrm{kHz}$ from the LHC beam crossing rate of $40 \mathrm{MHz}$, where $\sim 20 \%$ is allocated for electrons and photons $(\mathrm{e} / \gamma)$. This two-level system is composed in first place, of a Level1 hardware-based trigger (L1), that reduces the rate from $40 \mathrm{MHz}$ to $100 \mathrm{kHz}$, using low granularity data from calorimeters and the muon system, and identifies regions-of-interest (RoIs). Then, the software-based high-level trigger (HLT) performs the online particle reconstruction, using full granularity data from all of the detector in addition to the RoIs from accepted events by the L1.

The increased energy and luminosity plus the challenging pile-up conditions of the LHC in Run-2 made it necessary to optimize the techniques used and the trigger selections, to control the rates while keeping high efficiencies for processes of interest. It is important to mention that the instantaneous luminosity is two times the designed maximum of $10^{34} \mathrm{~cm}^{-2} \mathrm{~s}^{-1}$.

This document summarizes the ATLAS electron and photon trigger in Run-2, discussing the implementation of new Neural Network techniques implemented for online electron selection, areas where improvement is still possible and needed, and the performance of the trigger in the early 2018 data taking.

\section{Triggering $e / \gamma$}

In the L1 trigger, electron and photon candidates are selected using the energy deposition in the electromagnetic (EM) calorimeter, provided they pass $\eta$-dependent transverse energy $\left(E_{\mathrm{T}}\right)$ threshold and hadronic isolation requirements.

The HLT $e / \gamma$ triggers reconstruct objects within EM RoI provided by the L1 Calorimeter (L1Calo) trigger. At the HLT, a sequence of $e / \gamma$ reconstruction and identification algorithms are executed where fast algorithms are used first, to reject background events early and precise algorithms are used later, to efficiently identify $e / \gamma$, with cut-based identification (ID) for photons and Likelihood-based ID for electrons. In this sequence, the calorimeter clusters are reconstructed with a sliding window algorithm, and the selection is based on the $E_{\mathrm{T}}$ of the cluster. Common shower shape and energy ratios variables for electrons and photons are calculated for identification. Photons are reconstructed using only EM calorimeter clusters while electrons are reconstructed using both, EM calorimeter clusters and tracking information. The electron candidates need to have tracks loosely matched to the cluster based on $(\Delta \phi, \Delta \eta)$ and satisfy requirements using hits in the tracking detectors and transition radiation hit information. 


\section{Ringer Algorithm}

The Ringer algorithm is a multivariate discriminator, an ensemble of neural networks, using information from concentric rings in the calorimeter, designed to have the same signal efficiency (figure 1) and a high rejection power ( $2-3 \mathrm{X})$ compared to the previously used cut-based selection. It is build using all calorimeter layers, centered in a window around the cluster barycenter. The first ring in each layer is the cell closest to cluster barycenter, the next ring is the collection of cells around the previous one, where the ring value is the sum $E_{\mathrm{T}}$ of all cells composing the ring. The primary chain latency reduces from $200 \mathrm{~ms}$ to $\sim 100 \mathrm{~ms}$, and in the full set of electron and photon triggers the latency reduction is $\sim 1 / 4$. The Ringer is only applied for triggers with $E_{\mathrm{T}}$ thresholds $\geq 15 \mathrm{GeV}$, the cut-based method is still applied for lower $E_{\mathrm{T}}$ trigger, but studies are on-going in order to be able to only use Ringer-based selections.

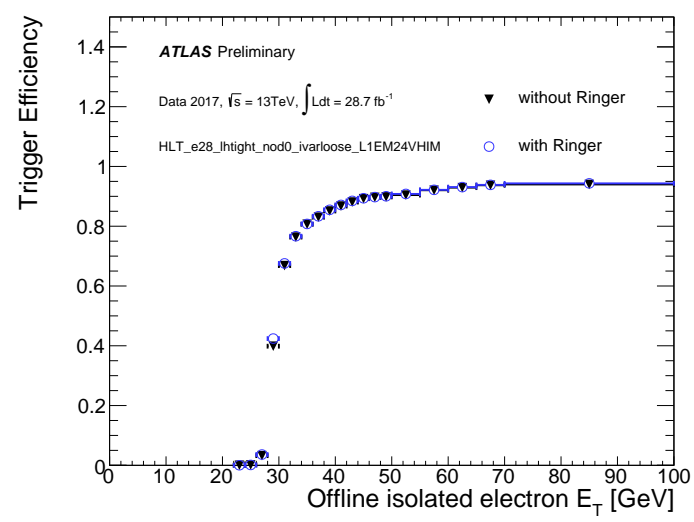

Figure 1: Electron trigger efficiency with and without Ringer [3], measured with a sub-set of 2017 data, as a function of the offline reconstructed electron candidate's $E_{\mathrm{T}}$.

\section{Electron and Photon HLT trigger rates and performances}

The output HLT rates for different photon and electron triggers as a function of the instantaneous luminosity for the full 2016 dataset are shown in figure 2. Both electron and photon trigger rates have a roughly linear dependence on the instantaneous luminosity.

The electron efficiencies are measured with a tag-and-probe method, using $\mathrm{Z} \rightarrow$ ee decays in early 2018 data and late 2017 data, where the offline reconstructed electrons are required to pass a likelihood-based ID.

The efficiencies of an electron trigger requiring $E_{\mathrm{T}}>28 \mathrm{GeV}$, a likelihood-based tight identification and a loose isolation are shown in figure 3 as a function of $E_{\mathrm{T}}$ and the average interactions per crossing $(\langle\mu\rangle)$. The error bars show statistical uncertainties. The performance achieved in 2018 conditions is the same as in 2017, showing to be stable and robust against pileup.

The photon efficiencies are measured based on a data-driven bootstrap (BS) method, using events selected from a fully efficient reference trigger to measure the performance of 

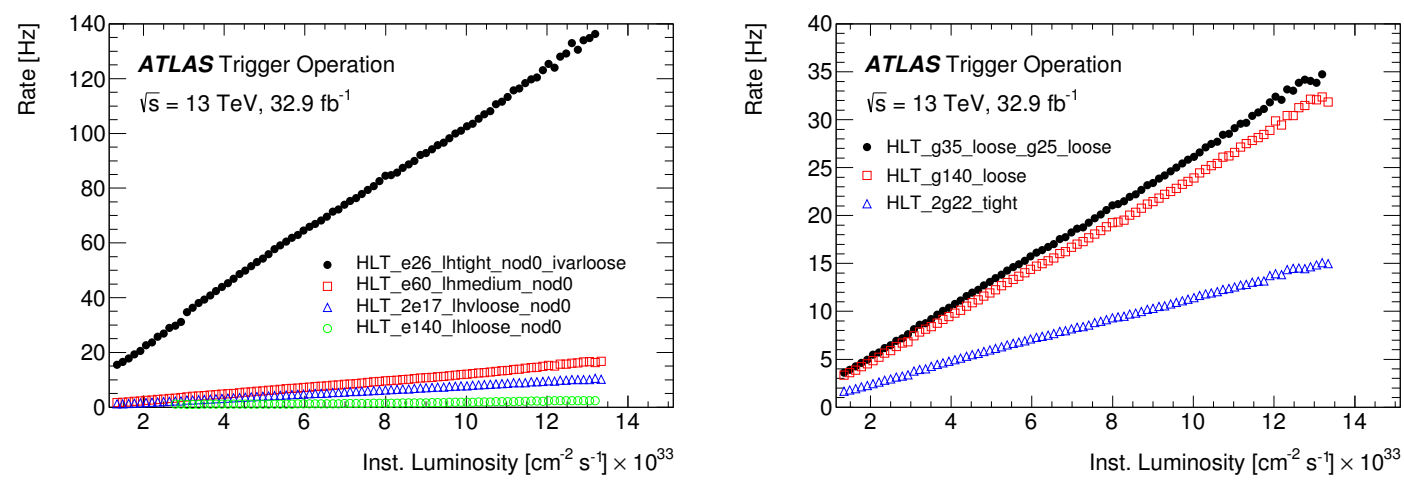

Figure 2: Output rates of the single-electron and di-electron primary triggers (left) and the photon primary triggers (right), as a function of the un-calibrated instantaneous luminosity measured online during the 2016 proton-proton data taking at a center-of-mass energy of $13 \mathrm{TeV}$ [3].
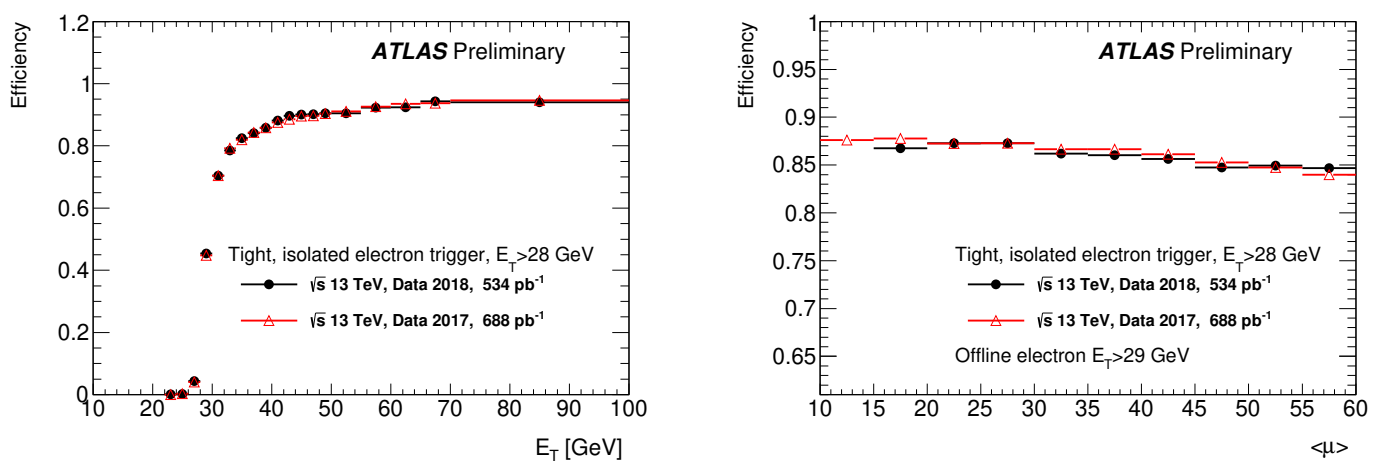

Figure 3: Efficiency of the tight, isolated electron trigger with $E_{\mathrm{T}}>28 \mathrm{GeV}$, as a function of $E_{\mathrm{T}}$ (left) and $\langle\mu\rangle$ (right), in $534 \mathrm{pb}^{-1}$ of 2018 data (black circles) and in $688 \mathrm{pb}^{-1}$ of 2017 data (red triangles) as a reference [3].

the offline trigger. The photon ID is done with a cut-based selection. The efficiency of a photon trigger requiring $E_{\mathrm{T}}>25 \mathrm{GeV}$, a medium cut-based ID and no isolation are shown in figure 4 as a function of $E_{\mathrm{T}}$ and $\langle\mu\rangle$. No background subtraction is applied to the BS sample. The trigger performance is very close to $100 \%$ from $E_{\mathrm{T}} \sim 5 \mathrm{GeV}$ above the trigger threshold. In addition, the efficiency is constant and robust against pileup.

\section{Sources of inefficiency for single electron triggers}

The trigger developments are made to minimize the differences between online and offline selection. In order to know where there are still improvements needed, the sources of inefficiencies for single electron triggers, are studied using data 2017, at each selection step in the HLT with respect to the offline reconstruction. Figure 5 shows a list of the differences observed, that are mainly in the precision electron step, in particular in the likelihood discriminant.

\section{Conclusions}

Triggering electrons and photons with a high efficiency is a key part of the ATLAS 

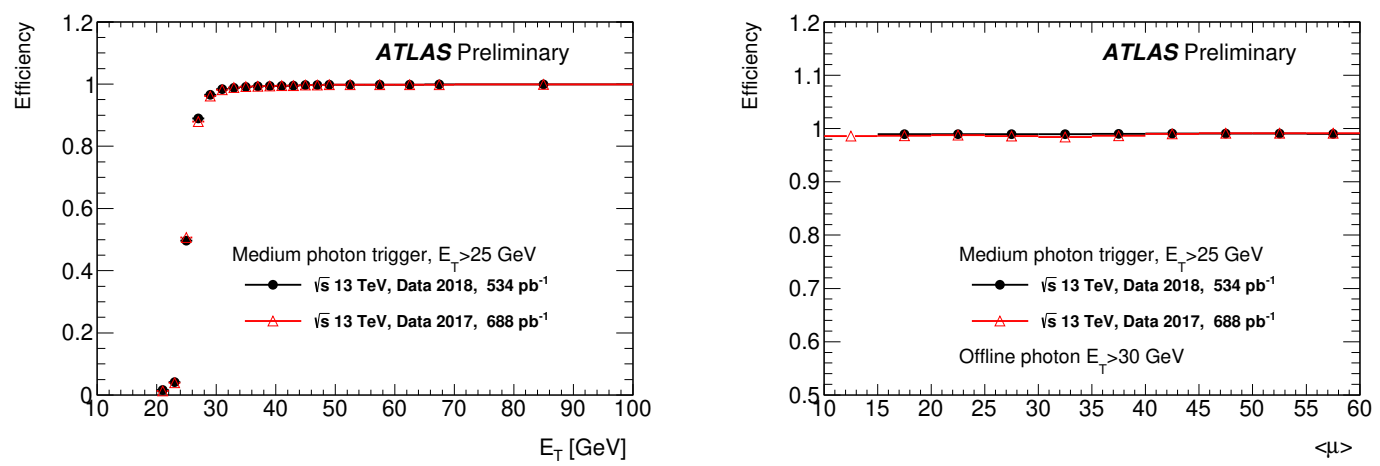

Figure 4: Efficiency of the medium photon trigger with $E_{\mathrm{T}}>25 \mathrm{GeV}$, as a function of $E_{\mathrm{T}}$ (left) and $<\mu>$ (right), in $534 \mathrm{pb}^{-1}$ of 2018 data (black circles) and in $688 \mathrm{pb}^{-1}$ of 2017 data (red triangles) as a reference [3].

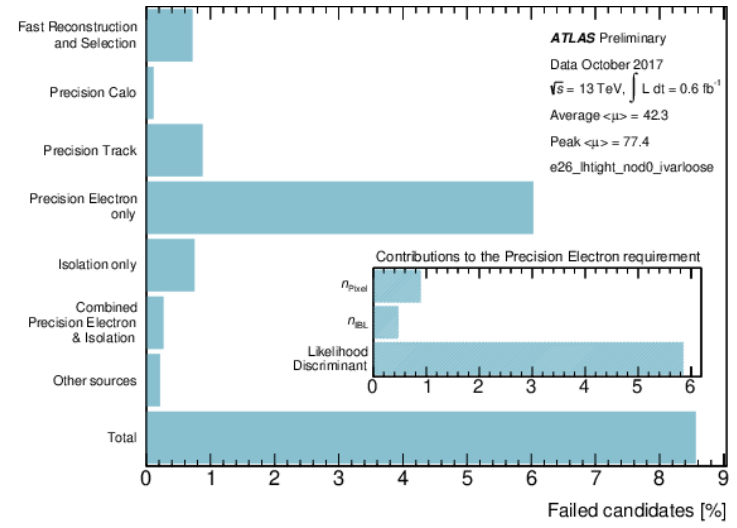

Figure 5: Sources of inefficiency for the tight, isolated electron trigger with $E_{\mathrm{T}}>26 \mathrm{GeV}$, at each selection step in the HLT with respect to the offline reconstruction and the corresponding L1 requirements in a data run taken in October 2017 [3].

data taking process. The challenging conditions of the LHC Run-2 require a constant optimization and improvement of the trigger selection and the techniques used in order to keep the rates below limits while providing efficiently $e / \gamma$ objects for physics analysis. The ATLAS trigger system has proven to perform as expected fulfilling these requirements during the Run-2 data taking, even in the high luminosity conditions that are two times the designed value.

\section{References}

[1] ATLAS Collaboration, 2012, Eur. Phys. J. C72 1849 [hep-ex/1110.1530].

[2] ATLAS Collaboration, 2008 JINST 3 S08003.

[3] ATLAS Collaboration, Public Egamma Trigger Plots. https://twiki.cern.ch/twiki/bin/view/AtlasPublic/EgammaTriggerPublicResults [ATL-COM-DAQ-2018-005, ATL-COM-DAQ-2018-049, ATL-COM-DAQ-2018-007]. 